\title{
Review
}

\section{Validation Studies for Microbial Contamination and Control of Contaminants}

\author{
HIDEHARU SHINTANI
}

\begin{abstract}
Faculty of Science and Engineering, Chuo-University, 1-13-27, Kasuga, Bunkyo, Tokyo 112-8551, Japan
\end{abstract}
Received 29 October, 2014/Accepted 30 June, 2015

\begin{abstract}
Microbiological control is a regulatory requirement and one that can be defined as the continued interaction of science and applied technology with products, processes, materials, equipment, and personnel entering the manufacturing areas. In the ISO 14644-1, control of microbial contamination is addressed.

A good microbiological control program starts with understanding the risks for microbial contamination in the manufacturing process and identification of possible types of contaminants. The results obtained from such risk assessment can be used in the design of facilities and equipment as well as when establishing protocols regarding equipment and personnel. Once possible sources of contamination have been identified, control and preventative measures can be implemented and qualified/validated.
\end{abstract}

Key words : Microbial contamination / Disinfectant / Contaminant.

\section{INTRODUCTION}

Microbial contamination is costly to companies in terms of equipment damage, production downtime, product contamination investigations, and energy losses. Recently, there has been a paradigm shift in the pharmaceutical and biotechnology industries to move away from the conventional approach of dealing with microbial contamination in a reactive mode to a proactive approach that includes understanding the process and ensuring quality by design. Companies now seek to understand the sources of contaminants, environmental conditions, and facility and equipment design that can lead to microbial colonization and proliferation. In addition, companies have started to evaluate alternate strategies for sanitization and cleaning verification to ensure that sanitization and disinfection procedures are effective. A better understanding of how microorganisms survive and proliferate in the manufacturing environment is also needed so that companies can implement effective microbial control strategies.

Microbial contamination control is not simply a task. It is a continuous effort involving all parts of the facility, all aspects of the process, and company personnel. It is

*Corresponding author. Tel: +81-4-2592-2336, E-mail : hshintani(a)jcom.zaq.ne.jp indeed an activity that truly involves continuous improvement. One that requires the support of company management and that must be embraced by manufacturing operators involved in the production of pharmaceutical drug products.

\section{RISK ASSESSMENT}

The principle of risk assessment as a tool to improve pharmaceutical processes was introduced as the announcement of an initiative called Pharmaceutical cGMPs [FDA, 2004]. This initiative was designed to enhance and modernize the regulation of pharmaceutical manufacturing and product quality through the implementation of based on understanding the process principles, risk management in manufacturing, regulatory inspections, and the practice of decision-making based on sound scientific principles. With this initiative, the FDA created an atmosphere for change in the pharmaceutical industry, a shift in paradigm with the focus on understanding the process and implementing new technologies.

Process risk assessment tools such as Failure Mode and Effect Analysis [FMEA] and Hazard Analysis Critical Control Point [HACCP] have been successfully used by pharmaceutical companies to identify areas in the process and types of raw materials and equipment 
that are at high risk of being contaminated with microorganisms (WHO, 2003).

HACCP is a systematic, proactive, and preventative tool to identify, assess, and prevent or reduce potential risks that can occur at specific steps in a process.

Through the risk analysis process, critical control points are identified and monitored (Schothorst, 2004). Because microbial contamination can be introduced into the process through raw materials and excipients, clean utilities, equipment (design and flow), facilities (design, materials of construction, ventilation/air filtration systems, temperature, and humidity), and personnel, these medium-to-high-risk areas should be thoroughly evaluated and, whenever applicable, appropriate controls should be put into place. For example, because people area major source of microbial contamination in a manufacturing environment, companies should focus on effective aseptic technique training and cleanroom behavior and establish personnel flows and maximum numbers of people for their various manufacturing suites. Some other considerations during a HACCP assessment should include:

-Possibility of survival/proliferation of organisms in the product

-Potential for contaminants to produce toxins/toxic products

-Equipment-cleaning and sanitization procedures

-Facility-cleaning and sanitization procedures

-Evaluation of personnel involvement with the process

- Open versus contained/closed processes

-Processing time limits (holding times of in-process materials)

Establishing production time limits should be considered during process validation studies as a tool to control and prevent microbial proliferation in the product being manufactured. Time limits for the completion of each phase of a production run must be established and followed, whenever appropriate. For example, if a company implements a practice to hold a bulk drug product for an extended period of time before filling into the final containers, a holding time limit must be established to prevent microbial proliferation, thus ensuring the microbial quality of the final product. Typically, as part of a product hold-time validation study, bioburden/ microbial limit testing is performed at time zero and then at the end of the storage period.

Indeed, through problem-solving/risk analysis techniques and validation studies designed on the basis of sound scientific principles, pharmaceutical companies can create innovative approaches and develop effective procedures to prevent microbial contamination of drug products. Some of the critical programs at a pharma- ceutical company that have a direct impact in the microbial quality of the products manufactured are:

- Testing of raw materials and product samples for bioburden

-Cleaning/disinfection of facilities

-Equipment cleaning/validation studies

-Monitoring of the facilities, environment, and personnel

-Microbiological testing and validation of water systems

\section{OBJECTIONABLE ORGANISMS}

In pharmaceutical microbiology, there is a need and interest to screen for the presence of microorganisms that are objectionable to the process and products manufactured. Recently, there were four organisms of concern listed in the USP. They are Staphylococcus aureus, Pseudomonas aeroginosa, Escherichia coli, and Salmonella spp. and products were screened for the presence of one or more of these microbial species. These microorganisms are known pathogens and, when present in a product sample, often indicate the potential presence of similar organisms of concern. However, over the years it became evident that many pharmaceutical products were contaminated with microorganisms other than the four species listed above, and many of them could not be recovered using the given compendial methods used to screen for these bacterial species. Therefore, the regulatory agencies started to enforce the use of suitable methods for the screening of microorganisms that are known to be objectionable to a particular product or process. Method suitability is indeed one of the main concerns these days in pharmaceutical microbiology because compendial methods are designed for the recovery of culturable clinical isolates and often fall short of addressing the needs for the recovery of stressed and environmental isolates.

Although the regulatory agencies expect companies to be diligent about potentially objectionable microbes in their products, they do not tell a company which organisms they should screen for. Instead, the onus is on the pharmaceutical company to decide which organisms are objectionable and implement programs for product and raw material quality control testing accordingly. The decision on whether an organism is objectionable or not is up to the drug manufacturers, because the definition of objectable is product and process dependent.

Microorganisms may be deemed objectionable based on several factors, and often the decision is made on a case-by-case basis and using a risk-based approach. Some organisms may not be pathogenic or opportunistic pathogens, but can adversely impact the quality of 
the product being manufactured. As a guideline, some of the factors to be taken into consideration when deciding whether a given microbe should be deemed objectionable or not include:

-Type of microbial species

-Numbers of microorganisms isolated

-Product dosage form

- Intended product use

- Target patient population

-Route of administration

If the presence of a particular type of microorganism has the potential to adversely affect product quality (including stability) and safety, then the organism should be deemed objectionable. As such, the manufacturing company should implement appropriate screening tests for raw materials and product samples. Likewise, microorganisms that have the potential to adversely affect the integrity of the product container closure system (e.g., fermenting organisms that create gaseous pressures) and/or the bioavailability of the API should also be deemed objectionable.

Most microorganisms found in a pharmaceutical manufacturing environment are Gram-positive bacilli and Gram-positive cocci (generally human-borne types), yeasts, and filamentous fungi (mold); many of these types of isolates are nonpathogenic and therefore not considered objectionable. However, some of these microbes, such as Staphylococcus epidermidis, can become opportunistic pathogens for patients with weak immune systems. Gram-negative organisms are generally found in aqueous environments (e.g., water systems) and raw materials of natural origin. These types of organisms are usually pathogenic and produce toxins such as endotoxins (lipopolysacchrides in the cell wall of Gram negative bacteria, Figures 1 and 2). Bacterial endotoxins cause pyrogenic (fever) reactions. Therefore, products with direct contact with the blood

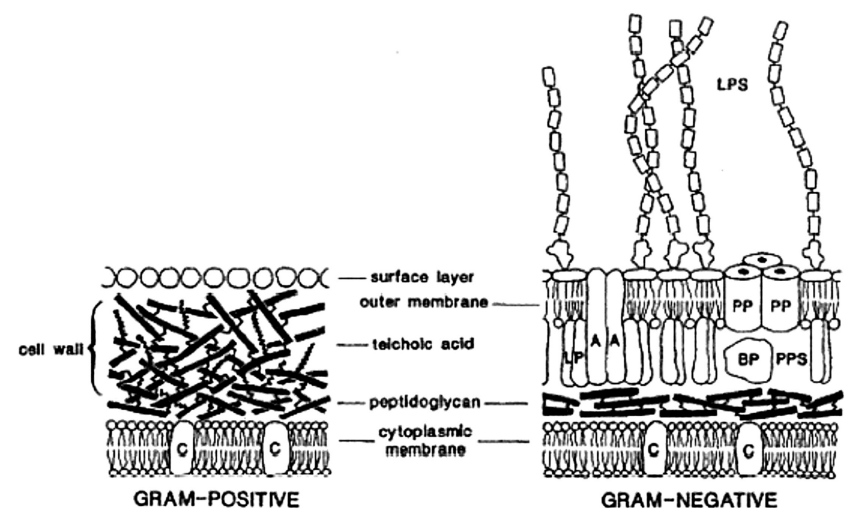

FIG. 1. Schematic diagram of the Gram-positive and Gramnegative cell envelope. A: outer membrane protein, BP: binding protein, C: cytoplasmic membrane-embedded protein, LPS:lipopolysaccharide. PP: porin, PPS: periplasmic space.

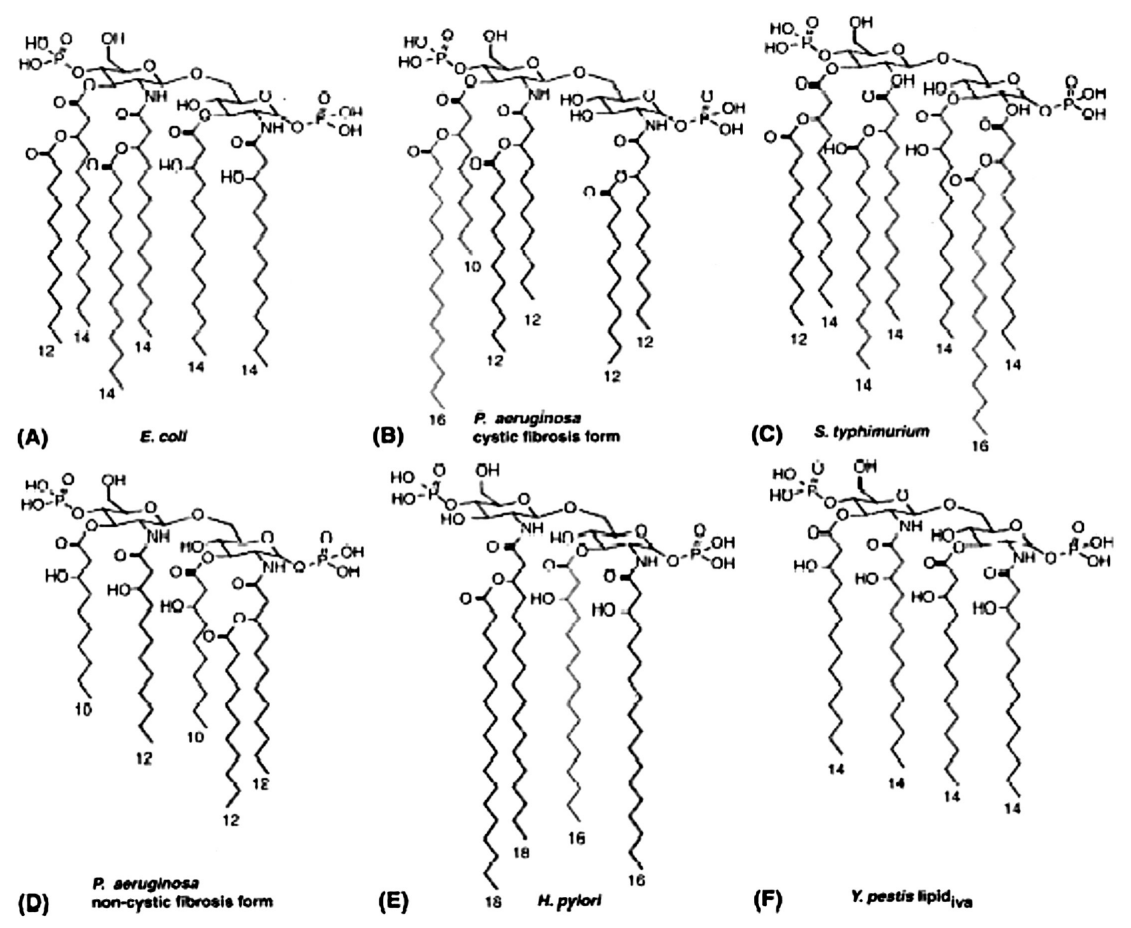

FIG. 2. The structural diversity of lipid A in Gram-negative microorganisms. 
stream (e.g., injectables, wound ophthalmics and topicals) should have specifications for bacterial endotoxin concentrations.

Cundel (2002) published a paper on food-borne pathogenic microorganisms (bacteria, viruses, and parasites) and natural toxins. In this paper it is listed the following known pathogenic bacteria: Salmonella spp, Clostridium botulinum, S. aureus, Campylobacter jejuni, Yersinia enterolytica, Yersinia pseudotuberculosis, Listeria monocytogenes, Vivrio cholerae OI Vibrio vulnificus, Clostridium perfringens, Bacillus cereus, Aeromonas hydrophila, Plesimonas shigelloides, Shigella spp. and Streptococcus spp. Also included is the enterovirulent Escherichia coli (Eec) group that includes Escherichia coli-enterotoxigenic (ETEC), Escherichia coli-enteropathogenic (EPEC), and Escherichia coli O157: H enterohemorrhagic (EHEC), and Escherichia coli-enteroinvasive (EIEC). Other organisms that have also been found responsible for human disease and infection include Aeromonas spp, Arcanobacterium haemolyticum, Aspergilus spp., Bacillus spp., Bacteroides fragilis, Bordetella pertussis, Burkholderia cepacia, Candida albicans, Chlamydia pneumoniae, Corynebacterium diphtheria, Cryptococcus spp., Enterococcus spp., Helicobacter pylori, Klebsiella pneumoniae, Microsporum spp., Moraxella catarrhalis, Mycobacterium turbeculosis, Mycoplasma pneumoniae, Neisseria meningitidis, Neiserria gonorrhoeae, Nocardia spp., Proteus mirabilis, Psedomonas spp., Serratia marcescens, Staphylococcus epidermidis, and Trychophyton spp. It seems that with the increase in the number of people with weak immune systems and microorganisms that have developed resistance to antimicrobials, the list of organisms of concern continues to grow.

\section{SANITIZATION AND DISINFECTION PRACTICES}

Sanitization and disinfection practices should be part of a microbial control program. Pharmaceutical products are at risk of microbial contamination during the manufacturing process, and therefore, procedures must be in place to ensure the microbial quality of the manufacturing environment. The USP Chapter $<1072>$ provides an useful information on the selection of chemical disinfectants and antiseptics and the demonstration of their antimicrobial efficacy (disinfectant qualification studies). It also addresses the application of disinfectants in sterile pharmaceutical manufacturing as well as regulation and safety considerations.

The control of microbial contamination at manufacturing facilities is addressed via a company's cleaning and disinfection procedures, which include physical and chemical methods for removal and destruction of microbes. Physical means of cleaning are capable of removing particles and debris that can harbor microbial cells. Chemical sanitizers and disinfectants reduce microbial contamination by inactivating microorganisms that might be present in the environment.

Chemical agents that destroy microorganisms are classified as sanitizers, disinfectants and sporicides. Products that kill only bacteria are referred to as bacteriocidal, whereas agents that kill only fungi are referred to as fungicidal. Chemical agents that do not completely kill the microbes and only inhibit their proliferation are referred to as static agents; chemicals that inhibit bacterial growth are referred to as abacteriostatic, and products that inhibit fungal growth are referred to as fungistatic. Chemical products that are capable of inactivating all types of microorganisms including bacterial spores, are referred to as sporicides or chemical sterilants.

In this review article, the use and qualification of disinfectants and sporicides as they apply to the contamination control of pharmaceutical-manufacturing facilities and equipment are discussed.

\section{Definitions and Types of Chemical Products Sanitizers}

Sanitizers are chemical agents capable of reducing the number of viable bacteria by $99.999 \%$ in 30 s under specific test conditions (Block, 1991). These types of products have limited antimicrobial activity and are unable to inactivate bacterial spores. Sanitizers also cannot handle soil and should therefore be applied only to precleaned surfaces. Examples of typical sanitizers used in the pharmaceutical industry are $70 \%$ isopropyl ethanol (IPA) and 70\% ethanol. Given the fact that alcohols do not leave residues, these chemicals are widely used for sanitization of product contact and work surfaces despite their limited antimicrobial properties.

\section{Disinfectants}

Disinfectants are chemical agents that kill vegetative forms of infectious bacteria. These chemicals have greater antimicrobial efficacy in comparison to sanitizers. Disinfectants are able to achieve 100\% reduction in the number of microbial contaminants as estimated by the AOAC International 10-min use-dilution test, with the exception of bacterial spores and filamentous fungi (Block, 1991). Unlike sanitizers, most disinfectants available on the market are capable of handling soil, and therefore they do not have to be applied only to precleaned surfaces. Typical disinfectant chemicals used in the pharmaceutical industry for cleaning of facilities include phenolic-based compounds, quaternary ammonium compounds, sodium hypochlorite, 
aldehydes, peracetic acid, and hydrogen peroxide. The antimicrobial efficacy of these compounds and the level of residue that is left on surfaces after application vary. Antimicrobial effectiveness is dependent on chemical formulation, concentration, and use; some of these products can achieve reduction of filamentous fungi (mold) and bacterial spores. Although most disinfectants and their residues are somewhat corrosive, the presence of disinfectant residues on surfaces provide for continued antimicrobial protection. Therefore, disinfectant manufactuers recommend rinsing chemical residues only in cases where significant buildup becomes an issue or to prevent corrosion of certain types of surfaces; understanding the chemistry of the disinfectants is critical so one can best recommend their use and application.

\section{Sporcides}

Sporcidides are chemical compounds that are capable of destroying all types of organisms jncluding bacterial spores (Block, 1991). Because bacterial spores are more resistant than vegetative cells (Table 1), a sporicidal agent is considered a sterilant. These types of products are extremely corrosive to stainless steel, plastic, and soft metals, and can be a health hazard to operators. It is common practice to remove chemical residues with sterile 70\% IPA to reduce or prevent corrosion. Most sporicidal agents are also not capable of handling soil, and thus, must be applied to precleaned surfaces. Examples of liquid sporicides widely used in the pharmaceutical industry include hydrogen peroxideperacetic acid blends, acidified ( $\mathrm{pH}$ 5-6) sodium hypochlorite (bleach) solutions (typical concentration:
1000-2000 ppm), and so on. Although sporicidal agents provide for the greatest reduction in microbial contamination, their use is selective, limited to remediation events, or as an alternate product as part of a disinfectant rotation program. Other products such as chlorine dioxide, formaldehyde, peracetic acid, and hydrogen peroxide, all in gaseous form, have been used in the pharmaceutical industry as fogging agents for decontamination of closed environments (e.g., manufacturing suites and isolator systems). The hydrogen peroxide vapor technology is an example of chemical sterilizing system widely used for decontamination of isolators, workstations, filling lines, and rooms. These systems provide for rapid antimicrobial activity without leaving residues.

\section{Factors in Choice and use of Disinfecants}

Careful attention must be given to the selection, preparation, storage, and application of disinfectants to ensure maximum efficacy. Most disinfectant products are produced as liquid concentrates that must be diluted with water before use. Therefore their preparation and storage are critical to ensure the quality of the disinfectant solution that will be applied to surfaces. There are several types of products on the market, and the user must carefully evaluate which disinfectants would best suit their facility sanitization/cleaning needs. Some products may not be compatible with one another and therefore would not be suitable for rotation; others may not be compatible with the surfaces that need to be decontaminated; certain chemicals may not be allowed in certain countries; and some products may not be the right formulation for the application. The following is a

TABLE 1. Order of tolerance of microorganisms to disinfectant

\begin{tabular}{|c|c|c|}
\hline & Microorganism & Examples \\
\hline \multirow{12}{*}{$\begin{array}{c}\text { More } \\
\text { Resistant }\end{array}$} & Prions & Scrapie, Creutzfeldt-Jakob disease, Chronic wasting disease \\
\hline & Bacterial spores & Bacillus, Geobacillus, Clostridium \\
\hline & Protozoal oocysts & Cryptosporidium \\
\hline & Helminth eggs & Ascaris, Enterobius \\
\hline & Mycobacteria & Mycobacterium tuberculosis, M. terrae, M. chelonae \\
\hline & Small, nonenveloped viruses & Poliovirus, Parvoviruses, Papillomaviruses \\
\hline & Protozoal cysts & Giardia, Acanthamoeba \\
\hline & Fungal spores & Aspergillus, Penicillium \\
\hline & Gram-negative bacteria & Pseudomonas, Providencia, Escherichia \\
\hline & Vegetative fungi and algae & Aspergillus, Trichophyton, Candida, Chlamydomonas \\
\hline & Vegetative helminths and protozoa & Ascaris, Cryptosporidium, Giardia \\
\hline & Large, nonenveloped viruses & Adenoviruses, Rotaviruses \\
\hline \multirow{2}{*}{$\begin{array}{c}\text { Less } \\
\text { Resistant }\end{array}$} & Gram-positive bacteria & Staphylococcus, Streptococcus, Enterococcus \\
\hline & Enveloped viruses & Human immunodeficiency virus, Hepatitis B virus, Herpes simplex virus \\
\hline
\end{tabular}


list of key factors that should be considered when choosing a disinfectant:

-Compatibility of surfaces with disinfectant

- Operator safety

- Need for residual antimicrobial activity

-Population and types of microbial contaminants that must be eradicated

- Chemical quality, sterility, and stability

- Cleaning ability of the disinfectant

- Vendor support

- Cost and availability

-Regulations

Other factors that must be considered to ensure maximum effectiveness in the control of microbial contamination include:

-Vendor's directions must be followed as written.

- Grade of water to prepare dilutions should be process grade.

-Disinfectant dilutions must be kept in clean containers and stored for defined and validated periods of time.

- Products used in Grade A and B areas should be sterile (EC 2003, FDA Guideline for Industry 2004).

- $\mathrm{pH}$ of disinfectant solution should be monitored since antimicrobial activity is dependent on and affected by $\mathrm{pH}$.

-Contact time of disinfectant with the surface to be decontaminated is critical and should be confirmed during disinfectant qualifications studies.

- Temperature of the diluent used in the preparation of the disinfectant solution and ambient temperature of area to be decontaminated can affect disintectant performance. Antimicrobial activity of the disinfectant is often slower when applied to cold rooms; therefore, a longer contact time may be required.

- Organic matter present on surfaces may diminish the antimicrobial effectiveness of certain chemicals not designed to handle soil.

-Disinfectant residues should be removed only to control buildup and prevent/reduce corrosion.

\section{Rotation of Disinfectants}

The practice of rotating disinfectants as a means of proactively eradicating a broad spectrum of microorganisms that may be present in a facility is nowadays a common practice in the pharmaceutical industry as well as a regulatory expectation. In other authority (EC 2003), it is stated that "where disinfectants are used, more than one type should be employed. Monitoring should be undertaken regularly in order to detect the development of resistant strains." The practice of rotation of disinfectants is also mentioned in the FDA guide for aseptic processing (FDA Guideline for Industry, 2004).

Rotation of disinfectants has been and continues to be a highly debated topic with many experts on opposite sides of the debate; some state that they have scientific data to prove the need for rotation, whereas others back up their justification for not rotating disinfectants with more data. One fact that is easy for all to agree on is the real and current regulatory expectation and enforcement of rotation of disinfectants. However, regulators do not stipulate the types of disinfectants to be used or the frequency with which these chemicals should be used. It is up to each company to evaluate its manufacturing facilities and, depending on environmental monitoring data, establish a sound cleaning/disinfection program that should include the alternate use of chemical agents with a varying and broad spectrum of antimicrobial activity.

\section{QUALIFICATION OF DISINFECTANTS}

Besides rotation, there are other regulatory expectations regarding the use of disinfectants that should be considered by the user; these include vendor qualification, procedures for product acceptance and rejection, and in-house disinfectant qualification and disinfectant requalification studies. Disinfectants marketed are regulated by the authorities, and administered by the EPA in USA. Chemical sterilizers that are intended for use on critical or semicritical medical devices are regulated by the FDA. According to these regulations, disinfectant manufacturers are required to supply information on product use dilution, types of microorganisms killed, and contact times required. Disinfectant manufacturers must also ensure their products meet established standards for efficacy and safety. Efficacy of disinfectants is verified by the vendor according to test methods established by the AOAC. As a user, a company is expected to perform additional studies to evaluate the effectiveness of the disinfectants as they are prepared and used and to evaluate the storage conditions of disinfectant dilutions for possible loss of efficacy over time. These studies are important because the efficacy of a disinfectant can be affected by types of surfaces, contact time, method of application, and type of microbial flora present. In fact, users are expected to perform challenge tests on disinfectants not only with standard test organisms but also with facility environmental isolates because commercially available microorganisms behave quite differently from their "wild" counterparts. Selection of the test organisms is crucial and an important issue to the regulatory agencies, especially when it comes to environmental isolates.

The test protocol used in a disinfectant qualification 
study varies from company to company because methods must be customized to reflect the types of surfaces and application of the chemical products at a particular facility. In general, two types of tests are performed: in situ and in vitro testing.

Disinfectant effectiveness studies are inadequate if:

- Only in vitro studies have been conducted.

- NO product contact surface or equipment studies have been conducted.

- Organisms used in the in vitro studies failed to include yeast and/or mold.

-No expiration dating studies have been conducted for opened disinfectants.

In this article, the current industry practices and regulatory expectations in terms of disinfectant qualification protocols are discussed.

\section{In Situ Testing}

In situ testing is performed to evaluate the effectiveness of the cleaning/disinfection procedures. Typically these studies are carried out by monitoring the manufacturing facilities before and after routine cleaning takes place and over several days (a minimum of three consecutive days is recommended). Monitoring in worstcase conditions (e.g., after a shutdown), when there is the potential for greater number and types of microbes in the environment, provides for better assessment of the disinfection efficacy. During these studies the number of sample sites (surface and air samples) is increased and the types of activities in the area are documented. The EM data collected before and after cleaning are compared. A typical criterion for acceptance in in situ studies requires EM data after cleaning to be below the established alert levels for the given area. In situ studies add value to the overall assessment of cleaning/disinfection effectiveness by providing realworld results from the proper use and application techniques of the chemical agents and personnel practices. However, a disinfectant qualification study is not complete unless in vitro studies are also conducted to confirm the actual antimicrobial effectiveness of the chemical agent against selected microorganisms.

\section{In vitro Testing}

There are three main types of studies performed in this category, and all are carried out in a laboratory setting because the test methods call for challenging the chemical agents with live cultures: the AOAC test method (Official Methods of Anlysis of AOAC International, 1999), surface challenge test (USP31-NF26a), and use-dilution test (Official Methods of Anlysis of AOAC International, 1999). Typical challenge organisms used
TABLE 2. Typical Test Organisms Used in Disinfectant Qualification Studies

\begin{tabular}{cl}
\hline Standard Challenge Organisms & Typical Environmental Isolates \\
\hline Escherichia coli & Micrococcus luteus \\
ATCC 11229 (AOAC) & Staphylococcus epidermidis \\
ATCC 8739 (USP) & Corynebacterium jeikeium \\
Staphylococcus aureus & Pseudomonas vesicularis \\
ATCC 6538 (AOAC, USP) & Rhodococcus globerulus \\
Pseudomonas aeruginosa & Pseudomonas fluorescens/putida \\
ATCC 15442 (AOAC) & Burkholderia cepacia \\
ATCC 9027 (USP) & Ralstonia pickettii \\
Bacillus subtilis (spore) & Bacillus sphaerricus \\
ATCC 19659 & Bacillus cereus/thuringiensis \\
ATCC 6633 (USP) & Paenibacillus spp. \\
Candida albicans & Penicillium chrysogenum \\
ATCC 10231 (AOAC, USP) & Aspergillus niger \\
ATCC 2091 (AOAC) & Alternaria spp. \\
Penicillium chrysogenum & Fusarium spp. \\
ATCC 11709 (AOAC) & Paecilomyces spp. \\
Aspergillus niger & \\
ATCC 16404 (AOAC, USP) & \\
\hline
\end{tabular}

for in vitro studies are presented in Table 2.

\section{AOAC test}

The AOAC hard surface carrier test is a qualitative method. The general study outline involves the use of stainless steel penicylinders inoculated with the test organisms (target load per dried carrier: 0.5-2.0 $\times 10^{6}$ CFU) and placed into test tubes containing the disinfectant solution to be evaluated. The contaminated carriers remain in contact with the disinfectant for a specified amount of time (contact time). Following the desired contact time, the carriers are removed, placed into a neutralizing medium, and incubated at specified conditions. Following incubation, the test tubes are observed for growth. Verification of the identity of the challenge organism recovered in the tubes with microbial growth is recommended. A standard performance evaluation at $95 \%$ confidence level for disinfectant activity is obtained by demonstrating the killing of each organism in 59/60 replicates tests. A product may be classified as a sporicidal agent if it kills each spore forming bacteria tested in 60/60 replicated trials. This AOAC method meets FDA and EPA criteria for disinfectant claims and is generally carried out by disinfectant manufacturers. As a user, a company may elect not to perform this test in favor of the surface test or use-dilution test, because the carrier test is very time consuming and requires 
skilled technicians with excellent aseptic and microbial manipulation techniques. In fact, most pharmaceutical companies no longer perform this test, which is not seen as a true requirement to demonstrate disinfectant efficacy at the user site. In the USP, it is stated that in order to demonstrate efficacy of a disinfectant within a pharmaceutical-manufacturing environment, it may be deemed necessary to conduct use-dilution tests, surface challenge tests, and in situ studies.

\section{Use-Dilution Test}

The use-dilution test, also referred to as a time-kill study, is derived from the AOAC use-dilution method. This test is quantitative and demonstrates log reduction of a wide range of test organisms (including environmental isolates) upon exposure to various disinfectant concentrations and at various contact use dilution times. The study outline typically involves inoculating an aliquot of the disinfectant with the test organism to achieve a concentration of approximately $10^{6} \mathrm{CFU} / \mathrm{mL}$. At selected time points (generally zero, $5 \mathrm{~min}$, and 10 min), aliquots of the inoculated disinfectant are removed, placed into a neutralizing medium, and selected dilutions plated (using the pour-plate method or membrane filtration method) with an agar medium for microbial enumeration purposes. Prepared plates are incubated at specified conditions appropriate for the test organism being evaluated. After incubation, recovered colonies are enumerated and log reductions are calculated based on the initial inoculum. Verification of the identity of the challenge organism is recommended. There is no standard test performance evaluation; however, most protocols designed by pharmaceutical companies require a minimum of 4-log reduction of vegetative bacteria and fungi in order to demonstrate disinfectant properties and a minimum of 6-log reduction in bacterial spores to demonstrate sporicidal activity.

\section{Surface Challenge Test}

Surface challenge tests are customized procedures based on the AOAC method for germicidal spray products and designed to evaluate the effectiveness of a disinfectant against standard and environmental isolates when applied to representative surfaces found in a manufacturing facility. This test has become the preferred disinfectant qualification method by the regulatory agencies. It is quantitative and demonstrates log reduction of the test organism upon exposure to the selected disinfectant concentration as it is used by a company during a cleaning procedure. In order to not deliberately contaminate the manufacturing areas, surface challenge tests are performed in a laboratory setting and use representative surfaces (referred to as coupons) that have been scaled down to a size of about $5 \times 5 \mathrm{~cm}$. Coupons used in surface challenge tests are often made from materials such as stainless steel, glass, vinyl, polycarbonate, plexiglass, epoxy-coated gypsum, and terrazzo tiles.

The typical study outline involves inoculating each of the test coupons with about $0.1 \mathrm{~mL}$ of an inoculum suspension of vegetative cells or spore suspension (target: $10^{6} \mathrm{CFU}$ to $10^{7} \mathrm{CFU} / 0.1 \mathrm{~mL}$ ). The inoculum is then spread evenly over the coupon and, depending on the test method it is dried onto the test coupon. Drying often results in loss of cell viability for vegetative organisms. Therefore, most companies either use a drying step only for bacterial spores or use a higher starting inoculum for vegetative organisms to account for loss in cell viability after drying. After inoculation (and drying if applicable), the chosen disinfectant solution is applied to the coupon surface and allowed to remain in contact with the test organisms for a specified amount of time (typical time points are zero, $5 \mathrm{~min}$, and $10 \mathrm{~min}$ ). The method of disinfectant application may vary depending on actual use at the manufacturing site; for example the disinfectant application may include spraying or wiping with a sterile wipe saturated with the disinfectant. It is important that the test protocol be designed to best mimic the company's cleaning procedure for the type of surface being evaluated.

Following the desired contact time the treated coupons are sampled for recovery of surviving organisms using swabs, rinse, or contact plates. Neutralizers that inactivate the disinfectants should be included in the diluent (swab and rinse method) and microbiological media should be chosen to ensure adequate recovery of viable cells. The aliquots of the test diluent that are plated with microbiological media or the contact plates are then incubated at specified conditions appropriate for the test organism being evaluated. At the end of incubation, the colonies recovered are enumerated and compared to the number of those from untreated inoculated coupons that have been extracted in the same manner (positive controls), and the log reduction in microbial population determined. Verification of the identity of the challenge organism is recommended. The standard performance evaluation for a surface challenge test is a minimum of 2-log reduction for bacterial spores and a 3-log reduction for vegetative organisms (bacteria and fungi) during the predetermined contact time (USP31-NF26a).

\section{Expiration Date for Disinfedant Solutions}

Companies must validate the expiration dates for prepared disinfectant solutions if they are not to be used on the day of preparation. These studies are performed to demonstrate that the diluted chemical agent 
will remain stable and active during the storage period and in the chosen container. The typical protocol outline for this type of study involves performing a use-dilution test on the day the disinfectant solution is prepared and once again at the end of the proposed expiry date. If there is a significant loss of efficacy compared to the initial result, i.e., greater than a 0.3-0.5 log variation, which is defined as the normal plating variability (USP31-NF26b), the company should not store the disinfectant for the proposed time frame and should consider a shorter storage period or an alternate storage container type.

\section{Sanitizers used for Equipment Cleaning}

Pharmaceutical-manufacturing equipment that cannot undergo steam-in-place (SIP) procedures or autoclaving must be chemically cleaned prior to use. Chemical sanitization can be accomplished using caustic, acidic, and oxidizing agents, such as hydrogen peroxide and sodium hypochlorite solutions. Chemical sanitization of equipment using cleaning-in-place (CIP) procedures provide for automatic cleaning and disinfecting of equipment without major disassembly and assembly of parts. A CIP cycle is more of a design method than a cleaning process, and it is achieved by placing pipes at an angle to the horizontal (minimum $3 \%$ ) to improve drainage, and using instruments and valves that connect flush to pipes, thereby eliminating "dead legs" in order to improve draining, to avoid biofilm formation and prevent stagnant pooling of liquid. In order to confirm the effectiveness of the chemical sanitization procedures, companies must perform studies that are similar to the use-dilution/time kill protocols performed for disinfectant qualification testing.

A typical study outline involves inoculating separate aliquots of the chemical sanitizer with various standard test organisms, including environmental isolates, to achieve a concentration of approximately $10^{6} \mathrm{CFU} / \mathrm{mL}$. At selected time points, depending on the equipment cleaning protocol, aliquots of the inoculated sanitizer are removed, placed into a neutralizing medium, and selected dilutions plated (using the pour-plate method or membrane filtration method) with an agar medium for microbial enumeration purposes. Prepared plates are incubated at specified conditions appropriate for the test organism being evaluated. After incubation, recovered colonies are enumerated and log reductions are calculated based on the initial inoculum. Verification of the identity of the challenge organism is recommended. There is no standard test performance evaluation. Most protocols for evaluation of microbiocidal properties are based on a minimum requirement of a 2-log reduction in spore-forming bacteria and a minimum 3-log reduction in vegetative bacteria (USP31-NF26a).
As with disinfectant qualification testing, traditional test protocols for evaluating the antimicrobial effectiveness of sanitizing and disinfectant solutions are established using ideal laboratory conditions with liquid cultures of free cells (planktonic cells). This allows for excellent and uniform physical contact of the antimicrobial agent and the microbial cells that are metabolically active. In cases where the equipment is operated at different temperatures or there is indication of biofilm formation in the equipment, the test results obtained from these traditional qualification studies become questionable.

\section{Neutralization and Microbial Recovery Studies}

In order to ensure the validity of the data obtained from in vitro disinfectant qualification testing, the study protocol must include neutralization and microbial recovery studies as test controls. Neutralization studies must be performed for each type of organism, disinfectant, and coupon (for surface test only) combination tested to demonstrate the ability of the medium to support growth of any viable organism.

Table 3 provides a list of typical chemical neutralizers that can be used in these studies. Some culture broths are formulated with a variety of neutralizing agents and are thus considered "universal neutralizing media." Dey/ Engley (D/E) is an example of a universal neutralizing broth widely used in disinfectant qualification studies. Microbial recovery studies are performed for each type of organism, disinfectant, and coupon (for surface test only) combination tested to ensure the extraction/recovery efficiency for the viable test organisms from the test surfaces/liquid medium (test-positive controls). Neutralization and microbial recovery studies are designed based on compendial methods for validation of microbial recovery.

\section{Requalification and Change Control}

Requalification of disinfectants and cleaning procedures is not required unless a significant change has been made to the program. The regulatory expectation is that facilities, systems, equipment, programs, and processes (to include cleaning and disinfection) should be periodically reviewed to confirm that they remain valid and in a state of control (EU, 2001). If a significant change has been made, a company should evaluate it via a change control program. For example if a company decides to change the types or manufacturers of qualified disinfectants/sanitizers, the proposed change must be first evaluated via a formal documentation system; sometimes, an annual review of the environmental monitoring data indicates an adverse trend or the presence of atypical organisms. In such cases, limited requalification of cleaning/disinfectant procedures may 
TABLE 3. Neuttralizing Agents for Common Antimicrobials

\begin{tabular}{ll}
\hline Antimicrobial compound & Potential Neutralizing Agents \\
\hline Alcohols & Dilution or polysorbate 80 \\
Aldehydes & Dilution or thiosultate \\
Bis-biguanide & Lecithin \\
Chlorhexidine & Polysorbate 80 and lecithin \\
EDTA & Mg $^{2+}$ and $\mathrm{Ca}^{2+}$ ions \\
Glutaraldehyde & Glycine and sodium bisumte \\
Halogens & Thiosultllte \\
lodine & Polysorbate \\
Mercuric chloride and other mercurials & Thioglycollate; Thiosulfate \\
Pafabens & Polysorbate 80 and lecithin \\
Phenolic compounds & Dilution or Polysorbate 80 and lecithin \\
Quatemary ammonium compounds & Polysorbate 80 and lecithin \\
Sodium hypochlorite & Sodium thiosulfate \\
Sorbates & Dilution \\
\hline
\end{tabular}

be required using the environmental strains that have demonstrated resistance to the existing cleaning procedures. In summary, periodic requalification/revalidation of approved procedures is not required as long as a company has routine monitoring and verification programs that are capable of detecting adverse trends and abnormal conditions.

\section{REFERENCES}

Ayorinde, J, O., Odeku, O. A., and Itiola, O. A. (2005) The Survival of Bacillus subtilis Spores in Dicalcium PhosPhate, Lactose, and Corn Starch and Their Binary Mixtures During Tableting, Pharmaceutical Technology, December 2, 2005.

Block, S. S. (1990) Disinfection, Sterilization, and Preservation, 4th ed., Malvern, PA, USA: Lea and Febiger.

Cundell, A. M. (2002) Comparison of Microbiological Testing Practices in Clinical, Food, Water and Pharmaceutical Microbiology in Relation to the Microbiological Attributes of Nutritional and Dietary Supplements, PharmacoPeial Forum, 28, [May-June 2002].

EC (2003) Guide to Good Manufacturing Practice, Revision to Annex 1 (2003), European Commission, Brussels, 30 May.

EU. (2001) Qualification and Validation, Final Version of Annex 15 to the EU Guide to Good Manufacturing Practice, European Commission, Brussels, July 2001.

FDA. (2004) Pharmaceutical cGMPs for the 21st Century Risk-Based Approach Final Report, Department of Health and Human Services, U. S. Food and Drug Administration.
FDA Guidance for Industry (2004) Sterile Drug Products Produced by Aseptic Processing-Current Good Manufacturing Practice, US Department of Health and Human Services, Food and Drug Administration, Center for Drug Evaluation and Research (CDER), Center for Biologics Evaluation and Research (CBER), Office of Regulatory Affairs(ORA), September 2004.

Hugo, W. B., and Russell, A. D., (1992) Pharmaceutical Microbiology, 5th ed. Boston, Blackwell Scientific Publications.

Obuekwe, I. F., Ogbimi, A. O., and Obuekwe, C. O. (2002) Microbial Contamination of Pharmaceutical Products in a Tropical Environment, Pakistan J. Sci. Industrial Research, 45, 341-344.

Official Methods of Analysis of AOAC International. (1999), 16th ed, 5th Revision, Baltimore, MD.

Van Schothorst, M. (2004) A Simple Guide to Understanding and Applying the Hazard Critical Control Point Concept, The Intemational Life Sciences Institute (ILSI) Europe Risk Analysis in Microbiology Task Force, 3rd ed.

USP31-NF26a, Chapter $<1072>$ Disinfectants and Antiseptics, United States Pharmacopeia, Baltimore, MD, USA.

USP31-NF26b, Chapters $<51>$, Antimicrobial Effectiveness Testing and $\langle 61\rangle$, Microbiological Examination of Non-sterile Products:Microbial Enumeration Tests.

WHO. (2003) Application of Hazard Analysis and Critical Control Point Methodology to Pharmaceuticals. In WHO Expert Committee on Specifications for Pharmaceutical Preparations, Thirty-Seventh Report, Geneva. World Health Organization, 2003, Annex 7 (WHO Technical Report Series, No.908). 